\title{
COINCIDENCE CLASSES IN NONORIENTABLE MANIFOLDS
}

\author{
DANIEL VENDRÚSCOLO
}

Received 15 September 2004; Revised 20 April 2005; Accepted 21 July 2005

We study Nielsen coincidence theory for maps between manifolds of same dimension regardless of orientation. We use the definition of semi-index of a class, review the definition of defective classes, and study the occurrence of defective root classes. We prove a semi-index product formula for lifting maps and give conditions for the defective coincidence classes to be the only essential classes.

Copyright (C) 2006 Daniel Vendrúscolo. This is an open access article distributed under the Creative Commons Attribution License, which permits unrestricted use, distribution, and reproduction in any medium, provided the original work is properly cited.

\section{Introduction}

In $[2,6]$ the Nielsen coincidence theory was extended to maps between nonorientable topological manifolds. The main idea to do this is the notion of semi-index (a nonnegative integer) for a coincidence set.

Let $f, g: M \rightarrow N$ be maps between closed $n$-manifolds without boundary. If we define $h=(f, g): M \rightarrow N \times N$ as usual, then we may assume that $h$ is in a transverse position, that is, the coincidence set $\operatorname{Coin}(f, g)=\{x \in M \mid f(x)=g(x)\}$ is finite and for each coincidence point $x$ there is a chart $\mathbb{R}^{n} \times \mathbb{R}^{n}=U \subset N \times N$ such that $(U,(f, g)(M) \cap U, \Delta N \cap$ $U$ ) corresponds to $\left(\mathbb{R}^{n} \times \mathbb{R}^{n}, \mathbb{R}^{n} \times 0,0 \times \mathbb{R}^{n}\right)$ (see [6] for details).

We say that two coincidence points $x, y \in \operatorname{Coin}(f, g)$ are Nielsen related if there is a path $\gamma:[0,1] \rightarrow M$ with $\gamma(0)=x, \gamma(1)=y$ such that $f \gamma$ is homotopic to $g \gamma$ relative to the endpoints. In fact, this is an equivalence relation whose equivalence classes are called coincidence classes of the pair $(f, g)$.

Let $x, y \in \operatorname{Coin}(f, g)$ belong to the same coincidence class and let $\gamma$ be a path establishing the Nielsen relation between them. We choose a local orientation $\mu_{0}$ of $M$ in $x$ and denote by $\mu_{t}$ the translation of $\mu_{0}$ along $\gamma(t)$.

Definition 1.1 [6, Definition 1.2]. We will say that two points $x, y \in \operatorname{Coin}(f, g)$ are $R$ related $(x R y)$ if and only if there is a path $\gamma$ establishing the Nielsen relation between them 
such that the translation of the orientation $h_{*} \mu_{0}$ along a path in the diagonal $\Delta(N) \subset$ $N \times N$ homotopic to $h \gamma$ in $N \times N$ is opposite to $h_{*} \mu_{1}$. In this case the path $\gamma$ is called graph-orientation-reversing.

Since $(f, g)$ is transverse, $\operatorname{Coin}(f, g)$ is finite. Let $A \subset \operatorname{Coin}(f, g)$, then $A$ can be represented as $A=\left\{a_{1}, a_{2}, \ldots, a_{s} ; b_{1}, c_{1}, \ldots, b_{k}, c_{k}\right\}$ where $b_{i} R c_{i}$ for any $i$ and $a_{i} R a_{j}$ for no $i \neq j$. The elements $\left\{a_{i}\right\}_{i}$ of this decomposition are called free.

Definition 1.2. In the above setup the semi-index of the pair $(f, g)$ in $A=\left\{a_{1}, \ldots, a_{s}\right.$; $\left.b_{1}, c_{1}, \ldots, b_{k}, c_{k}\right\}$ is the number of free elements $s$ denoted by $\mid$ ind $\mid(f, g ; A)$ of $A$.

This definition makes sense, since it does not depend on a decomposition (c.f. [2, 6]). Moreover the semi-index is homotopy invariant, it is well defined for all continuous maps, and if $U \subset M$ is an open subset such that $\operatorname{Coin}(f, g) \cap U$ is compact, we can extend this definition to that of the semi-index of a pair on the subset $U$, which is denoted by | ind $\mid(f, g ; U)$.

Definition 1.3. A coincidence class $C$ of a transverse pair $(f, g)$ is called essential if | ind $\mid(f, g ; C) \neq 0$.

In [5] Jezierski investigates whether a coincidence point $x \in \operatorname{Coin}(f, g)$ satisfies $x R x$. Such points can occur only when $M$ or $N$ are nonorientable, in which case they are called self-reducing points. This is a new situation (see [5, Example 2.4]) that cannot occur neither in the orientable case nor in the fixed point context.

Definition 1.4 [5, Definition 2.1]. Let $x \in \operatorname{Coin}(f, g)$ and let $H \subset \pi_{1}(M), H^{\prime} \subset \pi_{1}(N)$ denote the subgroups of orientation-preserving elements. We define

$$
\begin{aligned}
\operatorname{Coin}\left(f_{\#}, g_{\#}\right)_{x} & =\left\{\alpha \in \pi_{1}(M, x) \mid f_{\#}(\alpha)=g_{\#}(\alpha)\right\}, \\
\operatorname{Coin}^{+}\left(f_{\#}, g_{\#}\right)_{x} & =\operatorname{Coin}\left(f_{\#}, g_{\#}\right)_{x} \cap H .
\end{aligned}
$$

Lemma 1.5 [5, Lemma 2.2]. Let $f, g: M \rightarrow N$ be transverse and $x \in \operatorname{Coin}(f, g)$. Then $x R x$ if and only if $\operatorname{Coin}^{+}\left(f_{\#}, g_{\#}\right)_{x} \neq \operatorname{Coin}\left(f_{\#}, g_{\#}\right)_{x} \cap f_{\#}^{-1}\left(H^{\prime}\right)$ (in other words, if there exists a loop $\alpha$ based at $x$ such that $f \alpha \sim g \alpha$ and exactly one of the loops $\alpha$ or $f \alpha$ is orientation-preserving).

Definition 1.6. A coincidence class $C$ is called defective if $C$ contains a self-reducing point.

Lemma 1.7 [5, Lemma 2.3]. If a Nielsen class $C$ contains a self-reducing point (i.e., $C$ is defective), then any two points in this class are $R$-related, and thus

$$
\text { | ind } \mid(f, g ; C)= \begin{cases}0 & \text { if } \# C \text { is even } \\ 1 & \text { if } \# C \text { is odd }\end{cases}
$$




\section{The root case}

In [1] we can find a different approach to extend the Nielsen root theory to the nonorientable case. They use the concept of orientation-true map to classify maps between manifolds of the same dimension in three types (see also $[7,8]$ ).

Definition 2.1. A map $f$ is orientation-true if for each loop $\alpha \in \pi_{1}(M), f \alpha$ is orientationpreserving if and only if $\alpha$ is orientation-preserving.

Definition 2.2 [1, Definition 2.1]. Let $f: M \rightarrow N$ be a map of manifolds. Then three types of maps are defined as follows.

(1) Type I: $f$ is orientation-true.

(2) Type II: $f$ is not orientation-true but does not map an orientation-reversing loop in $M$ to a contractible loop in $N$.

(3) Type III: $f$ maps an orientation-reversing loop in $M$ to a contractible loop in $N$. Further, a map $f$ is defined to be orientable if it is of Type I or II, and nonorientable otherwise.

For orientable maps they describe an Orientation Procedure [1, 2.6] for root classes. This procedure uses local degree with coefficients in $\mathbb{Z}$. For maps of Type III the same procedure is possible only with coefficients in $\mathbb{Z}_{2}$. Then they define the multiplicity of a root class, that is an integer for orientable maps and an element of $\mathbb{Z}_{2}$ for maps of Type III.

Now if we consider the root classes of a map $f$ as the coincidence classes of the pair $(f, c)$ where $c$ is the constant map, we have.

Theorem 2.3. Let $f: M \rightarrow N$ be a map between closed manifolds of the same dimension, without boundary.

(i) If $f$ is orientable, then no root class of $f$ is defective.

(ii) If $f$ is of Type III, then all root classes of $f$ are defective.

Proof. If $f$ is orientable and $\alpha$ is a loop in $M, f \alpha \sim 1$ implies that $\alpha$ is orientationpreserving. On the other hand by Lemma 1.5, a coincidence class $C$ of the pair $(f, c)$ is defective if and only if there exists a point $x \in C$ and a loop $\alpha$ at $x$ such that $f \alpha \sim 1$ and $\alpha$ is orientation-reversing.

Now if $f$ is a Type III map, then there exists a loop $\alpha \in \pi_{1}\left(M, x_{0}\right)$ such that $\alpha$ is orientation-reversing and $f \alpha \sim 1$. Let $x \in \operatorname{Coin}(f, g)$ be a root. We fix a path $\beta$ from $x$ to $x_{0}$. Then $\gamma=\beta \alpha \beta^{-1}$ is a loop based at $x$, orientation-reversing and $f \gamma \sim 1$. Thus $x$ is a self-reducing root.

In fact [1, Lemma 4.1] shows the equality between the multiplicity of a root class and its semi-index.

Theorem 2.4. Let $M$ and $N$ be closed manifolds of the same dimension, without boundary such that $M$ is nonorientable and $N$ is orientable. If $f: M \rightarrow N$ is a map, then all essential root classes of $f$ are defective. 
Proof. There is no orientation-true maps from a nonorientable to an orientable manifold. If $f$ is a Type II map then by [1, Lemma 3.10] $\operatorname{deg}(f)=0$ and $f$ has no essential root classes. The result follows by Theorem 2.3.

We use the ideas of Theorem 2.3 to state.

Lemma 2.5. Let $f, g: M \rightarrow N$ be two maps between manifolds of the same dimension. If there exist a coincidence point $x_{0}$ and a graph-orientation-reverse loop $\alpha$ based in $x_{0}$ such that $f \alpha$ is in the center of $\pi_{1}\left(N, f\left(x_{0}\right)\right)$, then all coincidence points of the pair $(f, g)$ are self-reducing points.

Proof. Let $x_{1} \in \operatorname{Coin}(f, g)$. We fix a path $\beta$ from $x_{0}$ to $x_{1}$ and we will show that for the loop $\gamma=\beta^{-1} \alpha \beta$, the loops $f \gamma$ and $g \gamma$ are homotopic and $\gamma$ is orientation-reverse. In fact $f \gamma \sim g \gamma$ means $f \beta^{-1} \cdot f \alpha \cdot f \beta \sim g \beta^{-1} \cdot g \alpha \cdot g \beta$ hence $f \alpha \cdot\left(f \beta \cdot g \beta^{-1}\right) \sim\left(f \beta \cdot g \beta^{-1}\right) \cdot g \alpha$. The last holds, since the homotopy class of $f \alpha \sim g \alpha$ belongs to the centre of $\pi_{1}\left(N, f\left(x_{0}\right)\right)$. On the other hand $\gamma=\beta^{-1} \cdot \alpha \cdot \beta$ is orientation-reverse, since so is $\alpha$.

Corollary 2.6. Let $f, g: M \rightarrow N$ be two maps between manifolds of the same dimension such that $f_{\#}\left(\pi_{1}(M)\right)$ is contained in the center of $\pi_{1}(N)$. If $(f, g)$ has a defective class, then all classes of $(f, g)$ are defective.

In particular this is true for $\pi_{1}(N)$ commutative.

\section{Covering maps}

Let $M$ and $N$ be compact, closed manifolds of the same dimension, let $f, g: M \rightarrow N$ be two maps such that Coin $(f, g)$ is finite, and let $p: \widetilde{M} \rightarrow M$ and $q: \tilde{N} \rightarrow N$ be finite regular coverings such that there exist lifts $\tilde{f}, \tilde{g}: \widetilde{M} \rightarrow \tilde{N}$ of the pair $f, g:$

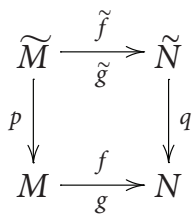

Under such hypotheses there is a bijection between the set of Deck transformations, $\mathfrak{D}(\widetilde{M})$, of the covering space $\widetilde{M}$ and the group $\left(\pi_{1}(M)\right) /\left(p_{\#}\left(\pi_{1}(\widetilde{M})\right)\right)$. We fix a point $\tilde{x_{0}} \in$ $\widetilde{M}$ and for each Deck transformation $\alpha$ we choose a path $\gamma$ in $\widetilde{M}$, from $\widetilde{x_{0}}$ to $\alpha\left(\widetilde{x_{0}}\right)$. Then, if $\bar{\alpha}$ is the projection of $\gamma$, the formula

$$
\mathfrak{D}(\widetilde{M}) \ni \alpha \longrightarrow[\bar{\alpha}] \in \frac{\pi_{1}\left(M, p\left(\widetilde{x_{0}}\right)\right)}{p_{\#}\left(\pi_{1}\left(\widetilde{M}, \tilde{x_{0}}\right)\right)}
$$

gives such bijection. It is easy to see that such bijection is an isomorphism of groups. 
The above isomorphism and a fixed lift $\tilde{f}$ determine the homomorphism from the $\operatorname{group} \mathfrak{D}(\widetilde{M})$ to $\mathfrak{D}(\tilde{N})$ for which the diagram

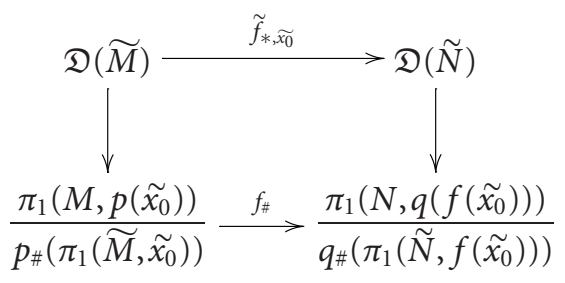

commutes. This homomorphism is given by the equality

$$
\tilde{f}_{*, x_{0}}(\alpha)(\tilde{f}(\tilde{x}))=\tilde{f} \alpha(\tilde{x}), \quad \forall \alpha \in \mathfrak{D}(\widetilde{M}), \forall \tilde{x} \in \widetilde{M} .
$$

The same construction can be done for map $g$ and we have the following.

Lemma 3.1. Let $\tilde{x_{0}} \in \operatorname{Coin}(\tilde{f}, \tilde{g})$ and $\alpha \in \mathfrak{D}(\widetilde{M})$. Then $\alpha\left(\tilde{x_{0}}\right) \in \operatorname{Coin}(\tilde{f}, \tilde{g})$ if and only if $\tilde{f}_{*, x_{0}}(\alpha)=\tilde{g}_{*, x_{0}}(\alpha)$ where $x_{0}=p\left(\tilde{x}_{0}\right)$.

Corollary 3.2. Let $\tilde{x_{0}} \in \operatorname{Coin}(\tilde{f}, \tilde{g})$ and $x_{0}=p\left(\tilde{x_{0}}\right)$. Then $p^{-1}\left(x_{0}\right) \cap \operatorname{Coin}(\tilde{f}, \tilde{g})$ have exactly \# Coin $\left(\tilde{f}_{*, x_{0}}, \tilde{g}_{*, x_{0}}\right)$ elements.

Lemma 3.3. Let $\tilde{x}_{0}$ and $\tilde{x}_{0}^{\prime}$ be two coincidences of the pair $(\tilde{f}, \tilde{g})$ such that $p\left(\tilde{x}_{0}\right)=p\left(\tilde{x}_{0}^{\prime}\right)=$ $x_{0}$, and let $\gamma$ be the unique element of $\mathfrak{D}(\widetilde{M})$ such that $\gamma\left(\tilde{x}_{0}\right)=\tilde{x}_{0}^{\prime}$. The points $\tilde{x}_{0}$ and $\tilde{x}_{0}^{\prime}$ are in the same coincidence class of $(\tilde{f}, \tilde{g})$ if and only if there exists $\bar{\gamma} \in \pi_{1}\left(M, x_{0}\right)$ such that

(i) $[\bar{\gamma}] \in\left(\pi_{1}\left(M, x_{0}\right)\right) /\left(p_{\#}\left(\pi_{1}\left(\widetilde{M}, \tilde{x}_{0}\right)\right)\right)$ corresponds to $\gamma$;

(ii) $f_{\#}(\bar{\gamma})=g_{\#}(\bar{\gamma})$.

Proof. $(\Rightarrow)$ If $\tilde{x_{0}}$ and $\tilde{x}_{0}^{\prime}$ are in the same coincidence class of $(\tilde{f}, \tilde{g})$, there exists a path $\beta$ from $\tilde{x_{0}}$ to $\tilde{x}_{0}^{\prime}$ establishing the Nielsen relation, (i.e., $\tilde{f} \beta \sim \tilde{g} \beta$ ).

Take $\bar{\gamma}=p \beta \in \pi_{1}\left(M, x_{0}\right)$. We can see that $[\bar{\gamma}]=\gamma$ and $f \bar{\gamma}=q \tilde{f} \beta \sim q \tilde{g} \beta=g \bar{\gamma}$, this means that $f_{\#}(\bar{\gamma})=g_{\#}(\bar{\gamma})$.

$(\Leftarrow)$ The lift $\tilde{\gamma}$ of $\bar{\gamma}$ starting at $\tilde{x}_{0}$ is a path from $\tilde{x}_{0}$ to $\tilde{x}_{0}^{\prime}$ establishing the Nielsen relation, (i.e., $\tilde{f} \tilde{\gamma} \sim \tilde{g} \tilde{\gamma}$ ).

If $\gamma$ is a loop in a manifold, we say that $\operatorname{sign}(\gamma)=1$ or -1 if $\gamma$ is orientation-preserving or orientation-reversing, respectively.

Corollary 3.4. In Lemma 3.3, if the points ${\tilde{x_{0}}}_{0}$ and $\tilde{x}_{0}^{\prime}$ are in the same coincidence class of $(\tilde{f}, \tilde{g})$, then ${\tilde{x_{0}}} R \tilde{x}_{0}^{\prime}$ if and only if $\operatorname{sign}\left(\tilde{f}_{*, x_{0}}(\gamma)\right) \cdot \operatorname{sign}(\gamma)=-1$. In this case, $x_{0}$ is a selfreducing coincidence point.

Proof. First we note that since $f_{\#}(\bar{\gamma})=g_{\#}(\bar{\gamma}), \tilde{f}_{*, x_{0}}(\gamma)=\tilde{g}_{*, x_{0}}(\gamma)$ and we have that $\operatorname{sign}\left(\tilde{f}_{*, x_{0}}(\gamma)\right) \cdot \operatorname{sign}(\gamma)=-1$ if and only if the paths $\bar{\gamma}$ and $\tilde{\gamma}$ in the proof of Lemma 3.3 are both graph orientation-reversing.

If we denote by $j_{x_{0}}$ the natural projection from $\pi_{1}\left(M, x_{0}\right)$ to $\mathfrak{D}(\widetilde{M})$ and by $\operatorname{Coin}\left(f_{\#}, g_{\#}\right)_{x_{0}}$ the set $\left\{\bar{\alpha} \in \pi_{1}\left(M, x_{0}\right) \mid f_{\#}(\bar{\alpha})=g_{\#}(\bar{\alpha})\right\}$, we have the following. 
COROLlary 3.5. If $x_{0}$ is a coincidence of the pair $(f, g)$, then the set $p^{-1}\left(x_{0}\right) \cap \operatorname{Coin}(\tilde{f}, \tilde{g})$ can be partitioned in $\left(\# \operatorname{Coin}\left(\tilde{f}_{*, x_{0}}, \tilde{g}_{*, x_{0}}\right)\right) /\left(\# j_{x_{0}}\left(\operatorname{Coin}\left(f_{\#}, g_{\#}\right)_{x_{0}}\right)\right)$ disjoint subsets, each of them with $\# j_{x_{0}}\left(\operatorname{Coin}\left(f_{\#}, g_{\#}\right)_{x_{0}}\right)$ elements all of them Nielsen related (therefore they are contained in the same coincidence class of the pair $(\tilde{f}, \tilde{g}))$. Moreover, no two points of different subsets are Nielsen related.

Lemma 3.6. Let $x_{0}, x_{1}$ be coincidence points in the same coincidence class of the pair $(f, g), \bar{\alpha}$ be a path from $x_{0}$ to $x_{1}$ establishing the Nielsen relation, $\tilde{x}_{0}, \tilde{x}_{0}^{\prime}$ coincidence points of the pair $(\tilde{f}, \tilde{g})$ such that $p\left(\tilde{x_{0}}\right)=p\left(\tilde{x}_{0}^{\prime}\right)=x_{0}$, and $\gamma$ the unique element of $\mathfrak{D}(\widetilde{M})$ such that $\gamma\left(\tilde{x}_{0}\right)=\tilde{x}_{0}^{\prime}$. If $\tilde{\alpha}$ and $\tilde{\alpha}^{\prime}$ are the two liftings of $\bar{\alpha}$ starting at $\tilde{x}_{0}$ and $\tilde{x}_{0}^{\prime}$ respectively then:

(i) $\tilde{\alpha}(1)$ and $\tilde{\alpha}^{\prime}(1)$ are coincidence points of the pair $(\tilde{f}, \tilde{g})$;

(ii) $\tilde{\alpha}(1)\left(\tilde{\alpha}^{\prime}(1)\right)$ is in the same coincidence class as $\tilde{x}_{0}\left(\widetilde{x}_{0}^{\prime}\right)$;

(iii) $p(\tilde{\alpha}(1))=p\left(\tilde{\alpha}^{\prime}(1)\right)=x_{1}$;

(iv) $\gamma(\tilde{\alpha}(1))=\tilde{\alpha}^{\prime}(1)$.

(v) If $\bar{\alpha}$ is a graph orientation-reversing-path (in this case $x_{0} R x_{1}$ ), then $\tilde{\alpha}$ and $\tilde{\alpha}^{\prime}$ are graph orientation-reverse-paths (in this case $\tilde{x}_{0} R \tilde{x}_{1}$ and $\left.\tilde{x}_{0}^{\prime} R \tilde{x}_{1}^{\prime}\right)$.

Proof. (i), (ii), and (iii) are known (we prove using covering space theory). To prove (iv) we notice that $\gamma(\tilde{\alpha}(0))=\gamma\left(\tilde{x_{0}}\right)={\tilde{x_{0}}}^{\prime}=\tilde{\alpha}^{\prime}(0)$ implies $\gamma(\tilde{\alpha}(1))=\tilde{\alpha}^{\prime}(1)$.

To prove (v), we use [2, Lemma 2.1, page 77].

Theorem 3.7. Let $M$ and $N$ be compact, closed manifolds of the same dimension, let $f, g$ : $M \rightarrow N$ be two maps, and let $p: \widetilde{M} \rightarrow M$ and $q: \tilde{N} \rightarrow N$ be finite coverings such that there exist lifts $\tilde{f}, \tilde{g}: \widetilde{M} \rightarrow \tilde{N}$ of the pair $(f, g)$. If $\widetilde{C}$ is a coincidence class of the pair $(\tilde{f}, \tilde{g})$, then $C=p(\widetilde{C})$ is a coincidence class of the pair $(f, g)$ and

$$
\mid \text { ind } \mid(\tilde{f}, \tilde{g} ; \widetilde{C})= \begin{cases}s \cdot k(\bmod 2) & \text { if } C \text { is defective; } \\ s \cdot k & \text { otherwise }\end{cases}
$$

where $s=\mid$ ind $\mid(f, g, C), k=\# j\left(\operatorname{Coin}\left(f_{\#}, g_{\#}\right)_{x_{0}}\right)$ and $x_{0} \in C$.

Proof. Since | ind | is homotopy invariant, we may assume that $\operatorname{Coin}(f, g)$ is finite. The fact that $C=p(\widetilde{C})$ is a coincidence class of the pair $(f, g)$ is known. We choose a point $x_{0} \in C$. Since Coin $(f, g)$ is finite, we can suppose $C=\left\{x_{1}, \ldots, x_{s} ; c_{1}, c_{1}^{\prime}, \ldots, c_{n}, c_{n}^{\prime}\right\}$ where each $x_{i}$ is free, and for all pairs $c_{j}, c_{j}^{\prime}$ we have $c_{j} R c_{j}^{\prime}$.

Now we choose paths $\left\{\bar{\alpha}_{i}\right\}_{i}, 2 \leq i \leq s ;\left\{\bar{\beta}_{j}\right\}_{j}$ and $\left\{\bar{\gamma}_{j}\right\}_{j}, 1 \leq j \leq n$ (see Figure 3.1) such that

(i) $\bar{\alpha}_{i}$ is a path in $M$ from $x_{1}$ to $x_{i}$ establishing the Nielsen relation;

(ii) $\bar{\beta}_{j}$ is a path in $M$ from $x_{1}$ to $c_{j}$ establishing the Nielsen relation;

(iii) $\bar{\gamma}_{j}$ is a graph-orientation-reversing path in $M$ from $c_{j}$ to $c_{j}^{\prime}$.

Assume that $C$ is not defective. We notice that $p^{-1}\left(\left\{c_{1}, c_{1}^{\prime}, \ldots, c_{n}, c_{n}^{\prime}\right\}\right) \cap \widetilde{C}$ splits into the pairs of points $\left\{\tilde{\gamma}_{j}^{r}(0), \tilde{\gamma}_{j}^{r}(1)\right\}$ where $\tilde{\gamma}_{j}^{r}$ is the lift of $\bar{\gamma}_{j}^{r}(0)$ starting from a point $\tilde{c}_{i}^{r} \in p^{-1}\left(c_{i}\right)$. By Lemma $3.6(\mathrm{v})$ the points $\tilde{\gamma}_{j}^{r}(0), \tilde{\gamma}_{j}^{r}(1)$ are $R$-related. For the same reason no two points 


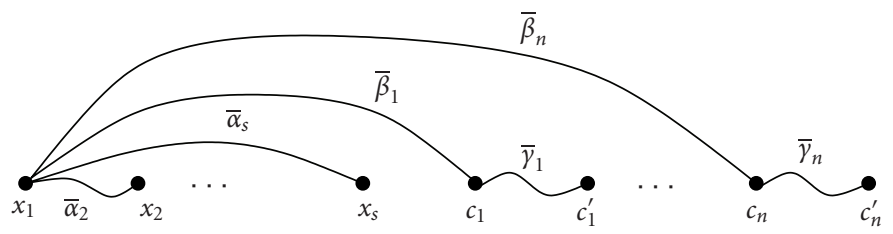

Figure 3.1. The class $C$ and the chosen paths.

from $p^{-1}\left(\left\{x_{1}, \ldots, x_{s}\right\}\right)$ are $R$-related. Thus

$$
\mid \text { ind }\left|(\tilde{f}, \tilde{g} ; \tilde{C})=\# p^{-1}\left(\left\{x_{1}, \ldots, x_{s}\right\}\right)=\right| \text { ind } \mid(f, g, C) \cdot k=s \cdot k .
$$

Now we assume that $C$ is defective. Then each point from $C$ is self-reducing hence so also is each point in $\widetilde{C}($ Lemma $3.6(\mathrm{v}))$. Now

$$
\begin{aligned}
\mid \text { ind } \mid(\tilde{f}, \tilde{g} ; \tilde{C}) & =\# \tilde{C}(\bmod 2) \\
& =k(s+2 n)(\bmod 2) \\
& =k \cdot s(\bmod 2) .
\end{aligned}
$$

\section{Twofold orientable covering}

Let $M$ and $N$ be compact closed manifolds of same dimension such that $M$ is nonorientable and $N$ is orientable; let $f, g: M \rightarrow N$ be two maps, and let $p: \widetilde{M} \rightarrow M$ be the twofold orientable covering of $M$. We define $\tilde{f}, \tilde{g}: \widetilde{M} \rightarrow N$ by $\tilde{f}=f p$ and $\tilde{g}=g p$ :

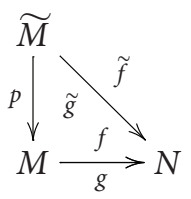

LEMMA 4.1. Under the above conditions, if $C$ is a coincidence class of the pair $(f, g)$, then $p^{-1}(C) \subset \operatorname{Coin}(\tilde{f}, \tilde{g})$ is such that

(1) $p^{-1}(C)$ can be divided in two disjoint sets $\widetilde{C}$ and $\widetilde{C}^{\prime}$, such that $p(\widetilde{C})=p\left(\widetilde{C}^{\prime}\right)=C$;

(2) if $\tilde{x_{1}}, \tilde{x_{2}} \in \tilde{C}$ (or $\left.\tilde{C}^{\prime}\right)$, then $\tilde{x_{1}}$ and $\tilde{x_{2}}$ are in the same coincidence class of $(\tilde{f}, \tilde{g})$;

(3) $\widetilde{C}$ and $\widetilde{C}^{\prime}$ are in the same coincidence class of the pair $(\tilde{f}, \tilde{g})$ if and only if $C$ is defective.

Proof. We make $q: \tilde{N} \rightarrow N$ as the identity map in the Corollaries 3.2, 3.4 and Lemma 3.6.

COROLlary 4.2. Under the hypotheses of Lemma 4.1 we have

(1) if $C$ is not defective, then $\widetilde{C}$ and $\widetilde{C}^{\prime}$ are two coincidence classes of the pair $(\tilde{f}, \tilde{g})$ such that $\operatorname{ind}(\tilde{f}, \tilde{g}, \widetilde{C})=-\operatorname{ind}\left(\tilde{f}, \tilde{g}, \widetilde{C}^{\prime}\right)$ and $|\operatorname{ind}(\tilde{f}, \tilde{g}, \widetilde{C})|=|\operatorname{ind}|(f, g, C)$; 
(2) if $C$ is defective, then $\widetilde{C} \cup \widetilde{C}^{\prime}$ is a unique coincidence class of the pair $(\tilde{f}, \tilde{g})$ with $\operatorname{ind}\left(\tilde{f}, \tilde{g}, \widetilde{C} \cup \widetilde{C}^{\prime}\right)=0$.

Proof. It is useful to remember that the pair $(\tilde{f}, \tilde{g})$ is a pair of maps between orientable manifolds and that ind $(\tilde{f}, \tilde{g}, \widetilde{C})$ are the indices of the coincidence class $\widetilde{C}$. Since the index and the semi index are homotopy invariants, we may assume that $\operatorname{Coin}(f, g)$ is finite.

(1) Since $M$ is nonorientable, the antipodism of $A: \widetilde{M} \rightarrow \widetilde{M}$, that is, the map exchanging the points in $p^{-1}(x)$ reverses the orientation of $\widetilde{M}$. On the other hand $A(\widetilde{C})=$ $\widetilde{C}^{\prime}$, hence ind $\left(\tilde{f}, \tilde{g} ; \widetilde{C}^{\prime}\right)=\operatorname{ind}(\tilde{f}, \tilde{g} ; A(\widetilde{C}))=\operatorname{ind}\left(\tilde{f} A^{-1}, \tilde{g} A^{-1} ; \widetilde{C}\right)=-\operatorname{ind}(\tilde{f}, \tilde{g} ; \widetilde{C})$.

(2) As above we deduce that for $\tilde{x}, \tilde{x}^{\prime} \in p^{-1}(x), \operatorname{ind}(\tilde{f}, \tilde{g} ; \tilde{x})=\operatorname{ind}\left(\tilde{f}, \tilde{g} ; \tilde{x}^{\prime}\right)$, hence ind $(\tilde{f}$, $\left.\tilde{g} ; p^{-1}(x)\right)=0$.

Corollary 4.3. Under de hypotheses of Lemma 4.1 we have

(1) $L(\tilde{f}, \tilde{g})=0$;

(2) $N(\tilde{f}, \tilde{g})$ is even;

(3) $N(f, g) \geq(N(\tilde{f}, \tilde{g})) / 2$;

(4) if $N(\tilde{f}, \tilde{g})=0$, then all coincidence classes with nonzero semi-index of the pair $(f, g)$ are defective.

Proof. We have that $p(\operatorname{Coin}(\tilde{f}, \tilde{g}))=\operatorname{Coin}(f, g)$, and in the pair $(\tilde{f}, \tilde{g})$ the pre-image, by $p$, of a defective class of the pair $(f, g)$ has index zero.

\section{Applications}

Theorem 5.1. Let $f, g: M \rightarrow N$ be two maps between closed manifolds of the same dimension such that $M$ is nonorientable and $N$ is orientable. Suppose that $N$ is such that for all orientable manifolds $M^{\prime}$ of the same dimension of $N$ and all pairs of maps $f^{\prime}, g^{\prime}: M^{\prime} \rightarrow N$ we have that $L\left(f^{\prime}, g^{\prime}\right)=0$ implies that $N\left(f^{\prime}, g^{\prime}\right)=0$. Then all coincidence classes with nonzero semi-index of the pair $(f, g)$ are defective.

Proof. The hypotheses on $N$ are enough to show, using the notation of the proof of Lemma 4.1, that $N(\tilde{f}, \tilde{g})=0$. So by Corollary 4.3 , all coincidence classes with nonzero semi-index of the pair $(f, g)$ are defective.

We notice that the hypotheses on the manifold $N$ in Theorem 5.1, in dimension greater than two, are equivalent to the converse of Lefschetz theorem. In dimension two these hypotheses are not equivalent but necessary for the converse of Lefschetz theorem.

Remark 5.2. The following manifolds satisfy the hypotheses on the manifold $N$ in Theorem 5.1:

(1) Jiang spaces [3, Corollary 1];

(2) nilmanifolds [4, Theorem 5];

(3) homogeneous spaces of a compact connected Lie group $G$ by a finite subgroup $K$ [3, Theorem 4]. 


\section{Acknowledgments}

This work was made during a postdoctoral year of the author at Laboratoire Émile Picard, Universite Paul Sabatier (Toulouse, France). We would like to thank John Guaschi and Claude Hayat-Legrand for the invitation and hospitality, Peter N.-S. Wong for helpful conversations, and the referee for his critical reading and a number of helpful suggestions. This work was supported by Capes-BEX0755/02-8 (International Cooperation CapesCofecub Project no. 364/01).

\section{References}

[1] R. F. Brown and H. Schirmer, Nielsen root theory and Hopf degree theory, Pacific Journal of Mathematics 198 (2001), no. 1, 49-80.

[2] R. Dobreńko and J. Jezierski, The coincidence Nielsen number on nonorientable manifolds, The Rocky Mountain Journal of Mathematics 23 (1993), no. 1, 67-85.

[3] D. L. Gonçalves and P. N.-S. Wong, Homogeneous spaces in coincidence theory, Matemática Contemporânea 13 (1997), 143-158, 10th Brazilian Topology Meeting (São Carlos, 1996), (P. Schweitzer, ed.), Sociedade Brasileira de Matemática.

[4] __ Nilmanifolds are Jiang-type spaces for coincidences, Forum Mathematicum 13 (2001), no. $1,133-141$.

[5] J. Jezierski, The semi-index product formula, Polska Akademia Nauk. Fundamenta Mathematicae 140 (1992), no. 2, 99-120.

[6] __ The Nielsen coincidence theory on topological manifolds, Fundamenta Mathematicae 143 (1993), no. 2, 167-178.

[7] P. Olum, Mappings of manifolds and the notion of degree, Annals of Mathematics. Second Series 58 (1953), 458-480.

[8] R. Skora, The degree of a map between surfaces, Mathematische Annalen 276 (1987), no. 3, 415423.

Daniel Vendrúscolo: Departamento de Matemática, Universidade Federal de São Carlos, Rodovia Washington Luiz, Km 235, CP 676, 13565-905 São Carlos, SP, Brazil

E-mail address: daniel@dm.ufscar.br 